\title{
CHEMOTHERAPY
}

\section{Rolapitant-a new and safer antiemetic agent}

Chemotherapy-induced nausea and vomiting (CINV) is one of the most feared adverse effects of chemotherapy. Now, three trials, all coordinated by Lee Schwartzberg and Bernardo Rapoport, show that rolapitant is a novel, safe and effective agent for the prevention of CINV.

CINV is divided into an acute phase (the first $24 \mathrm{~h}$ after treatment initiation) and a delayed phase (24-120 h after treatment); serotonin is the main mediator of the acute phase reactions, whereas those that occur in the delayed phase are mediated by neurokinin-1 (NK-1) and substance P. Thus, current treatment guidelines for the prevention of CINV recommend the use of an NK-1 receptor antagonist, a serotonin receptor antagonist and dexamethasone. However, most NK-1 receptor antagonists interact with CYP3A4 and, therefore, affect the pharmacokinetics of drugs administered concomitantly.

Rolapitant is an NK-1 receptor antagonist with high affinity for the human NK-1 receptor that does not interact with CYP3A4. Its half-life is around $180 \mathrm{~h}$ and thus a single dose might be sufficient to prevent CINV. The aim of three phase III trials was to assess the safety and efficacy of rolapitant in combination with granisetron (a $5-\mathrm{HT}_{3}$ receptor antagonist) and dexamethasone to prevent CINV induced by moderately emetic chemotherapy (MEC) and highly emetic chemotherapy (HEC). The MEC agents used were carboplatin, cyclophosphamide and irinotecan, among others. The HEC agents used were cisplatin, and anthracycline plus cyclophosphamide combinations.

In both the MEC and HEC settings, a significantly greater proportion of patients treated with rolapitant compared with placebo had no delayed-phase

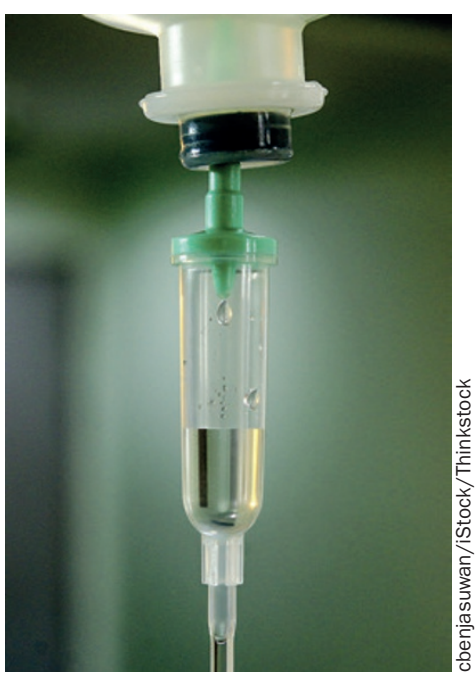

emesis, which addresses an unmet clinical need. In addition, patients receiving rolapitant who did not develop CINV in the acute phase were more likely to not develop CINV in the delayed phase.

"Rolapitant's long half-life offers us the opportunity to further explore dosing options-maybe it can be given the day before chemotherapy if that is more convenient for the patient," says Rapoport. Schwartzberg concludes, "patients often believe that they are supposed to vomit on chemotherapy, but the reality is we have very good drugs to prevent this. Some patients will stop their treatment because of their CINV and that's the last thing we want."

\section{Diana Romero}

Original articles Rapoport, B. L. et al. Safety
and efficacy of rolapitant for prevention
of chemotherapy-induced nausea and
vomiting after administration of cisplatin-
based highly emetogenic chemotherapy
in patients with cancer: two randomised,
active-controlled, double-blind, phase 3
trials. Lancet Oncol. doi:10.1016/S1470-
$2045(15) 00035-2$ | Schwartzberg, L. S.
et al. Safety and efficacy of rolapitant for
prevention of chemotherapy-induced nausea
and vomiting after administration of moderately
emetogenic chemotherapy or anthracycline
and cyclophosphamide regimens in patients
with cancer: a randomised, active-controlled,
double-blind, phase 3 trial. Lancet Oncol.
doi:10.1016/S1470-2045(15)00034-0

Original articles Rapoport, B. L. et al. Safety and efficacy of rolapitant for prevention of chemotherapy-induced nausea and vomiting after administration of cisplatinbased highly emetogenic chemotherapy in patients with cancer: two randomised active-controlled, double-blind, phase 3 trials. Lancet Oncol. doi:10.1016/S1470 2045(15)00035-2 | Schwartzberg, L. S. and vomiting after administration of moderately emetogenic chemotherapy or anthracycline and doi.10.1016/S1470-2045(15)00034-0 